FUNCIONES DE VOLUMEN, MODELOS DE CRECIMIENTO Y FACTOR DE FORMA PARA Drimys winteri Forst. Iván Quiróz Marchant, Ingeniero Forestal, División Regional, Instituto Forestal.

\title{
INTRODUCCION
}

Extensas áreas del territorio nacional se encuentran cubiertas por renovales de distintas especies forestales, generados como consecuencia de una intensa alteración de los bosques originales. En el caso específico de Canelo (Drymis winteri Forst), su capacidad colonizadora le ha permitido establecerse en forma pura o mezclada, predominando los bosques con desarrollo juvenil que se podrían calificar como renovales.

D. winteri es un árbol que se puede alcanzar alturas de 25 a $30 \mathrm{~m}$ (Donoso, 1978; Rodriguez et al., 1983) presenta tronco cilíndrico de hasta $1 \mathrm{~m}$ de diámetro con corteza gruesa, lisa, blanda y de color gris ceniciento (Hoffmann, 1982., Rodriguez et al., 1983).

Es una especie de amplia distribución geografica y de gran tolerancia a distintas condiciones ambientales. Crece junto a diferentes especies, formando parte de variadas asociaciones vegetales (Donoso, 1981).

La madera presenta escasa diferenciación entre albura y duramen, aunque este último es más oscuro, siendo de color castaño claro o amarillento-rosaceo. Los anillos de crecimiento son visibles, presentando la madera un vetado suave, brillante, debido a la presencia de radios leñosos de color café claro. Es una madera de peso medio, con densidades de 0,40 a 0,503 g/cm3 (Cuevas. 1983; Pérez, 1983).

Por el vetado la madera es muy apreciada para ebanistería, revestimiento de interiores, chapas, cajones e instrumentos musicales (Cuevas, 1983; Pérez, 1983). Es una especie adecuada para la fabricacion de tableros, superando sus características para este fin a las de Pinus radiata D. Don (Corvalán, 1987).

El largo de fibra de la especie destaca como el más alto entre las especies nativas e introducidas del país (Chesney, 1970; Rojas et al., 1975). Los requerimientos y condiciones para la cocción, consumo de reactivos y blanqueo en la fabricación de pulpa son similares a $\boldsymbol{P}$. radiata, obteniéndose además papeles de mayor resistencia a la tracción y similar resistencia al rajado (Rojas et al., 1975; Corvalán, 1987). 


\section{ANTECEDENTES GENERALES}

Las muestras utilizadas en el estudio fueron obtenidas en la Cordillera de la Costa en sus vertientes occidental y oriental, en $\operatorname{los} 72^{\circ} 18^{\prime}$ L.O., $39^{\circ} 46^{\prime}$ L.S. a 520 m.s.n.m. en exposición NE y $72^{\circ} 53^{\prime}$ L.O., $39^{\circ} 53^{\prime}$ L.S. a 750 m.s.n.m. y exposición N - NE, respectivamente.

\section{Clima}

De la estación meteorológica "Teja" la más cercana a la vertiente occidental, se obtuvieron temperaturas mínimas, medias y máximas de $7,9^{\circ} \mathrm{C}, 12,4^{\circ} \mathrm{C}$ y $17,5^{\circ} \mathrm{C}$ respectivamente, $78 \%$ de humedad relativa y $1.938 \mathrm{~mm}$ de precipitacion anual. La estacion meteorologica de "Punahue", representando a la vertiente oriental, registra valores medios de temperatura mínima, media y máxima de $5,9^{\circ} \mathrm{C}, 11,3{ }^{\circ} \mathrm{C}$ y $16,9^{\circ} \mathrm{C}$, respectivamente, con una precipitacion anual de $1.878 \mathrm{~mm}$ y un $85 \%$ de humedad relativa.

\section{Suelos}

Los suelos de la vertiente occidental de la Cordillera de la Costa son de tipo metamorfic ' mica - esquisto) con fuertes intrusiones graníticas. El análisis detallado del lugar de extracción de las muestras indica que éste tiene un suelo profundo a muy profundo, con un $\mathrm{PH}$ de fuertemente ácido a muy fuertemente ácido, con un contenido de materia orgánica de alto a muy alto. El contenido de $\mathrm{N}$ total es medio, la disponibilidad de $\mathrm{Mg}$ y $\mathrm{K}$ es media hasta los $35 \mathrm{~cm}$ variando a baja en profundidad y la disponibilidad de $\mathrm{P}$ y Ca es baja y alta, respectivamente.

La vertiente oriental de la Cordillera de la Costa presenta suelos delgados de 20 $-80 \mathrm{~cm}$, derivados generalmente de cenizas volcánicas depositadas sobre rocas metamorficas y depositos fluvio-glaciales. Determinaciones puntuales indican que el lugar presenta un suelo delgado con un $\mathrm{pH}$ fuertemente a muy fuertemente ácido; un contenido de materia orgánica de alto a muy alto y con un contenido de $\mathrm{N}$ total alto. La disponibilidad de $\mathrm{P}$ es baja y la de $\mathrm{K}$ y $\mathrm{Mg}$ es media hasta $\operatorname{los} 18 \mathrm{~cm}$, variando a baja en profundidad; la disponibilidad de $\mathrm{Ca}$ es alta hasta $\mathrm{los} 18 \mathrm{~cm}$, variando a media en profundidad.

\section{Características generales de los rodales muestreados}

Las características dasómetricas del renoval del sector occidental indican que éste tiene un área basal de $73 \mathrm{~m}^{2} /$ ha, constituida por 1.400 árboles por hectárea, con altura y diámetro de $21 \mathrm{~m}$ y $25 \mathrm{~cm}$ respectivamente, a la edad de 59 años. 
En tanto el sector oriental de la Cordillera de la Costa, el renoval se caracteriza por tener una área basal de $62 \mathrm{~m}^{2}$ con aproximadamente 2.700 árboles por hectárea, altura y diámetro promedio de $14 \mathrm{~m}$ y $17 \mathrm{~cm}$ respectivamente, a la edad de 46 años.

\section{METODOLOGIA}

En el estudio se utilizaron un total de 73 árboles, 38 del sector occidental y 35 del sector oriental de la Cordillera de la Costa. Este material proviene del raleo de parcelas permanentes instaladas por el Intituto Forestal (INFOR).

De éstos árboles se obtuvieron las variables de estado DAP, diámetro de copa, altura inicio de copa, altura total y la distancia a los cuatro árboles competidores más directos. En los árboles competidores, se midieron las mismas variables de estado anteriormente indicadas (Grosse y Cubillos, 1987).

La metodología empleada en la extracción y análisis de las rodelas para el análisis de tallo, fue la descrita por Kramer y Akca (1982) y Grosse y Cubillos (1987).

\section{Funciones de Volúmen}

Para la construcción de las funciones de volumen se utilizo el método de regresión paso a paso (Step-Wise). Los modelos utilizados fueron los propuestos por Zohrer (1980) para situaciones locales y regionales.

$$
\begin{aligned}
& \mathbf{V}=b_{0}+b_{1} D^{2} \\
& V=b_{0}+b_{1} D^{2} H \\
& V=b_{0}+b_{1} D^{3} \\
& V=b_{0}+b_{1} D^{2}+b_{2} H \\
& V=b_{0}+b_{1} D^{2}+b_{2} D^{2} H+b_{3} H
\end{aligned}
$$

Donde :

V : Volumen del árbol $\left(\mathrm{m}^{3} \mathrm{ssc}\right)$

D : Diámetro a $1,3 \mathrm{~m}(\mathrm{~cm} \mathrm{cc})$

H : Altura total (m)

$\mathrm{b}_{0}, \mathrm{~b}_{1}, \mathrm{~b}_{2}, \mathrm{~b}_{3}:$ : Coeficientes de regresion 


\section{Estimación del Incremento Diametral}

La elaboración del modelo de crecimiento diametral se basó en la metodología descrita por Cubillos (1987)

La variable dependiente del modelo, incremento diametral anual (IDAPA), se calculó con el valor medio del incremento diametral anual de los últimos 5 años de vida de cada árbol, estimando que durante este período la situación de competencia se mantendría constante (Grosse y Cubillos, 1987).

El modelo se construyó a través del análisis de regresión paso a paso, el cual consideró las siguientes variables :
DAP
H TOT
HINIC
Diámetro a la altura del pecho $(\mathrm{cm}$, medido a $1,3 \mathrm{~m})$
VOL TOT
Altura total (m)
DISME
Altura inicio de copa (m)
DAPCO
Volumen total $\left(\mathrm{m}^{3} \mathrm{ssc}\right)$
Distancia media a los árboles competidores (m)
H TO CO
DAP medio de los competidores
HINICO
Altura total promedio de los competidores (m)
DCCO
$\mathrm{E}$
Altura inicio copa promedio de los competidores (m)
IDAPA
Diámetro de copa medio de los competidores (m)
Edad (años)
Incremento periódico anual en diámetro (cm, media últimos
5 años).

\section{Factores de Forma}

Para la determinación del factor de forma natural se midió el diámetro a un décimo de la altura total y para el factor artificial a 1,3 m de altura. En la estimación de los factores de forma se probaron modelos lineales, exponenciales y múltiples, empleando como variables independiantes el DAP y la altura total.

\section{RESULTADOS}

La caracterización de las variables de estado de los árboles muestra competidores y del incremento diametral, involucradas en la construcción del modelo de crecimiento permite conocer el comportamiento de la muestra en relación a su disposición. 
Los bajos coeficientes de variacion obtenidos en el rodal sector occidental en las variables edad $(12,7 \%)$ y altura total $(8,5 \%)$ permiten considerarlo como coetaneo (Cuadro 1).

Cuadro 1: $\quad$ Estadística descriptiva de las variables de estado de los árboles muestra, competidores y crecimiento diametral. Sector occidental.

\begin{tabular}{|c|c|c|c|c|}
\hline \multirow{2}{*}{ VARIABLE } & \multicolumn{3}{|c|}{ VALORES } & \multirow{2}{*}{$\begin{array}{c}\text { COEFICIENTE } \\
\text { VARIACION } \\
(\%)\end{array}$} \\
\hline & MEDIO & MINIMO & MAXIMO & \\
\hline IDAPA & 0,18 & 0,08 & 0,54 & 48,6 \\
\hline DAP & 25,0 & 17,2 & 40,1 & 22,4 \\
\hline H. TOT & 21,2 & 17,5 & 26,5 & 8,5 \\
\hline H. FUST & 16,2 & 11,8 & 21,8 & 13,2 \\
\hline DC & 2,5 & 1,4 & 4,5 & 37,4 \\
\hline DAPCO & 27,1 & 17,0 & 43,1 & 22,4 \\
\hline H. TOT $C O(m)$ & 20,8 & 16,9 & 24,5 & 7,9 \\
\hline H. FUSCO (m) & 15,9 & 9,8 & 19,2 & 11,2 \\
\hline DISME (m) & 2,3 & 1,3 & 4,8 & 30,4 \\
\hline DCCO & 2,6 & 1,4 & 5,5 & 35,1 \\
\hline EDAD (аก๊os) & 58,5 & 43,0 & 79,0 & 12,7 \\
\hline
\end{tabular}

El renoval de canelo del sector oriental de la Cordillera de la Costa, también se considera coetáneo, por cuanto presenta un coeficiente de variación de $6,6 \%$ en la edad y $7,5 \%$ en la altura (Cuadro 2).

Cuadro 2: Estadística descriptiva de las variables de estado de los árboles muestra, competidores y crecimiento diametral. Sector oriental.

\begin{tabular}{|c|c|c|c|c|c|}
\hline \multirow{2}{*}{\multicolumn{2}{|c|}{ VARIABLE }} & \multicolumn{3}{|c|}{ VALORES } & \multirow{2}{*}{$\begin{array}{c}\text { COEFICIENTE } \\
\text { VARIACION } \\
(\%)\end{array}$} \\
\hline & & \multirow{2}{*}{$\begin{array}{r}\text { MEDIO } \\
0,21\end{array}$} & \multirow{2}{*}{$\begin{array}{r}\text { MINIMO } \\
0,08\end{array}$} & \multirow{2}{*}{$\frac{\text { MAXIMO }}{0,36}$} & \\
\hline IDAPA & $(\mathrm{cm})$ & & & & 36,7 \\
\hline DAP & (cm) & 16,5 & 10,7 & 21,7 & 18,8 \\
\hline Н. ТOT & (m) & 14,2 & 12,3 & 16,5 & 7,5 \\
\hline H. FUST & (m) & 9,9 & 7,0 & 12,5 & 14,2 \\
\hline & (cm) & 1,6 & 0,9 & 2,7 & 24,8 \\
\hline DAPCO & (cm) & 15,7 & 11,8 & 22,6 & 31,4 \\
\hline Н. ТОT CO & $(m)$ & 13,8 & 12,2 & 15,8 & 7,2 \\
\hline H. FUSCO & (m) & 10,1 & 6,7 & 19,5 & 20,9 \\
\hline DISME & (m) & 1,7 & 1,2 & 2,2 & 17,3 \\
\hline DCCO & (m) & 1,5 & 0,5 & 4,3 & 41,8 \\
\hline EDAD (an & años) & 46,0 & 41,0 & 55,0 & 6,6 \\
\hline
\end{tabular}




\section{Función de Volúmen}

En ambos sectores el mejor ajuste de la función volumen se logró con el modelo "australiano", dado que éste obtuvo la mayor correlación y el menor error standard. Los coeficientes " $b_{2}$ " de las funciones tienden a cero y por ende, no contribuyen a la función de volumen, razón por la cual no se les consideró.

Sector occidental

$$
\begin{aligned}
V & =-0,3767+0,0002 \mathrm{D}^{2}+0,0198 \mathrm{H} \\
\mathrm{r} & =0,97 \\
\text { Syx } & =5,0 \% \\
\mathrm{n} & =38
\end{aligned}
$$

Sector oriental

$$
\begin{aligned}
\mathrm{V} & =-0,1185+0,0005 \mathrm{D}^{2}+0,0091 \mathrm{H} \\
\mathrm{r} & =0,97 \\
\text { Syx } & =1,3 \% \\
\mathrm{n} & =35
\end{aligned}
$$

Donde :

$\mathrm{V}=$ Volumen del árbol $\left(\mathrm{m}^{3} \mathrm{ssc}\right)$ hasta $1 . U .10 \mathrm{~cm}$

$\mathrm{D}=$ Diámetro a 1,3 ( $\mathrm{cm} \mathrm{cc})$

$\mathrm{H}=$ Altura total (m)

\section{Modelo de Crecimiento Diametral}

El modelo de crecimiento diametral se construyo sobre la base de las variables independientes que mejor se correlacionaron con los indicadores del crecimiento. La mejor función de crecimiento para el sector occidental de la Cordillera de la Costa corresponde a :

$$
\begin{aligned}
\text { IDAPA } & =0,1496+0,0084 \text { DAP }-0,0031 \mathrm{E} \\
\mathrm{r} & =0,51 \\
\mathrm{Syx} & =7,4 \% \\
\mathrm{n} & =38
\end{aligned}
$$


El modelo de crecimiento diametral del sector oriental que mejor correlacion obtuvo, consideró el DAP del árbol de muestra y el DAP promedio de los competidores.

IDAPA $=0,1725+0,0128$ DAP - 0,111 DAPCO

$$
\begin{aligned}
r & =0,56 \\
\text { Syx } & =6,2 \% \\
n & =35
\end{aligned}
$$

Aún cuando estos modelos de crecimiento diametral no permiten obtener valores confiables, debido a su baja correlación, dan una idea del posible crecimiento que pueden lograr estos renovales.

Los modelos serán mejorados con la incorporación de nuevos antecedentes, provenientes de las parcelas permanentes, ubicadas en los sectores de donde se extrajo la información.

\section{Factores de Forma}

No se detectó ninguna tendencia clara del factor de forma natural o artificial en función del DAP-Altura. Por este motivo se optó por entregar valores medios según rangos diamétricos (Cuadro 3 y 4 ).

Cuadro 3: $\quad$ Factores de forma del sector occidental.

\begin{tabular}{|c|c|c|c|}
\hline \multirow{2}{*}{$\begin{array}{c}\text { RANGO } \\
\text { DIAMETRICO } \\
(\mathrm{cm})\end{array}$} & \multicolumn{2}{|c|}{ FACTOR DE FORMA } & \multirow{2}{*}{$\begin{array}{c}\text { NUMERO } \\
\text { DE }\end{array}$} \\
\cline { 2 - 3 } & ARTIFICIAL & NATURAL & OBSERVACIONES \\
\hline $16-20$ & 0,527 & 0,550 & 10 \\
$20,1-26$ & 0,505 & 0,566 & 14 \\
$26,1-40$ & 0,516 & 0,550 & 15 \\
\hline
\end{tabular}


Cuadro 4: $\quad$ Factores de forma del sector oriental

\begin{tabular}{|c|c|c|c|}
\hline \multirow{2}{*}{$\begin{array}{c}\text { RANGO } \\
\text { DIAMETRICO } \\
(\mathrm{cm})\end{array}$} & \multicolumn{2}{|c|}{ FACTOR DE FORMA } & \multirow{2}{*}{$\begin{array}{c}\text { NUMERO } \\
\text { DE } \\
\text { OBSERVACIONES }\end{array}$} \\
\hline & ARTIFICIAL & NATURAL & \\
\hline $\begin{array}{c}12-15 \\
15,1-19 \\
19,1-22\end{array}$ & $\begin{array}{l}0,622 \\
0,588 \\
0,571\end{array}$ & $\begin{array}{l}0,615 \\
0,592 \\
0,573\end{array}$ & $\begin{array}{l}16 \\
20 \\
15\end{array}$ \\
\hline
\end{tabular}

\section{BIBLIOGRAFIA}

CHESNEY, L. S. 1970. Aptitud papelera de Canelo (Drymis winteri Forst). Tesis Ing. Forestal. Santiago. Fac. Agronomía, U. de Chile 58 p.

CORVALAN, P. 1977. Estudio preliminar de crecimiento de algunos renovales de la Isla Grande de Chiloé. Tesis Fac. de Ciencias Forestales. U. de Chile. 106 p.

.1987 "El Canelo : una alternativa de desarrollo para la X Region" Santiago. Ministerio de Agricultura - Fac. Cs. Agrarias y Forestales, U. de Chile. Vol. II.

CUBILLOS V. (1987). Modelos de crecimiento diametral para algunos renovales de Raulí. Ciencia e Investigación Forestal (INFOR) № $1: 67$ - 77 p.

CUEVAS, E. 1983. Maderas nativas chilenas de interés en el comercio internacional. Investigacion y desarrollo forestal. Proyecto CONAF/PNUD/FAO-CHI/76/003 Documento de trabajo 48. $77 \mathrm{p}$.

DONOSO, C. 1978. Dendrología árboles y arbustos chilenos. $2^{\mathrm{a}}$ Edición Fac. Cs. Forestales, U. de Chile. Manual № 2142 p.

FAO $70 \mathrm{p}$.

1981. Tipos forestales de los bosques nativos de Chile. FO:DP/CH/76/003 CONAF-

GROSSE. H. Y CUBILLOS, V. 1987. Estudio de renovales de Raulí. Instituto Forestal. Chile. $124 \mathrm{p}$.

HOFFMANN, A. 1983. Flora Silvestre de Chile zona Austral. Santiago, Chile. Ed. Fundación Claudio Gay, 257 p. 
KRAMER, H. UND AKCA, A. 1982. Leitfăden für Dendrometrie und Bestandesinventur - J.D. Sauerländer's Verlag. Frankfurt a. M. 251 p.

PEREZ, V. 1983. Manual de propiedades físicas y mecánicas de maderas chilenas. Investigación y Desarrollo Forestal. Proyecto CONAF/PNUD/FAO-CHI/76/003. Documento de trabajo 47. $450 \mathrm{p}$.

RODRIGUEZ, R.; MATTHEI, S. Y QUEZADA, M. 1983. Flora arbórea de Chile. Concepción. U. de Concepción. 404 p.

ROJAS, M.; PISTONO, L. Y BLUMHMS, E. 1975. Densidad, largo de fibra y composicion de la madera de Canelo. INFOR. Informe Técnico 53.60 p.

SANCHEZ, C. 1986. Estructura y desarrollo de renovales puros y no intervenidos de Canelo (Drymis winteri J. et G. Forst) en la Isla de Chiloe. Tesis. Fac. de Ciencias Forestales. U. Austral de Chile. Valdivia. 110 p.

ZOHRER, F. 1980. Forstinventur. Verlag Paul Parey, Hamburg, Berlin. 207 p. 